Bull. Austral. Math. Soc.

$81 \mathrm{R} 10,17 \mathrm{~B} 37,16 \mathrm{w} 30$

Vol. 51 (1995) [177-194]

\title{
QUANTISED AFFINE ALGEBRAS AND PARAMETER-DEPENDENT $R$-MATRICES
}

\author{
Anthony J. Bracken, Mark D. Gould and Yao-Zhong Zhang
}

Let $U_{q}\left(\mathcal{G}^{(1)}\right)$ be a quantised non-twisted affine Lie algebra with $U_{q}(\mathcal{G})$ the corresponding quantised simple Lie algebra. Using the previously obtained universal $R$-matrices for $U_{q}\left(A_{1}^{(1)}\right)$ and $U_{q}\left(A_{2}^{(1)}\right)$, explicitly spectral-dependent universal $R$-matrices for $U_{q}\left(A_{1}\right)$ and $U_{q}\left(A_{2}\right)$ are determined. These spectral-dependent universal $R$-matrices are evaluated in some concrete representations; well-known results for the fundamental representations are reproduced, and an explicit formula for the spectral-dependent $R$-matrix associated with the $V_{(3)} \otimes V_{(\theta)}$ module is derived, where $V_{(3)}$ and $V_{(\theta)}$ carry the 3- and 6-dimensional representations of $U_{q}\left(A_{2}\right)$, respectively.

\section{INTRODUCTION}

Quantum deformations of universal enveloping algebras, or for short, quantum algebras, are perhaps amongst the most important discoveries in recent years in mathematics and theoretical physics $[8,10]$. The novelty of these algebras is that they have a quasitriangular Hopf algebra structure, that is to say each contains a canonical element $R$, called the universal $R$-matrix, which satisfies the spectral-independent quantum Yang-Baxter equation (QYBE). This equation plays a key role in applications to conformal field theories $[1,2]$ and knot theory $[20,23,24]$. Integrable models $[9$, $4,22]$, on the other hand, involve spectral-dependent $R$-matrices which satisfy the spectral-dependent QYBE.

Since the works of Jimbo and Jones $[11,13]$, a central problem has been the construction of spectral parameter-dependent $R$-matrices using quantum group techniques $[25,7,5]$. There are two commonly used procedures to this end in the literature: one is the so-called "Yang-Baxterisation" process, the other is the "fusion" method. By "Yang-Baxterisation" we mean two seemingly different but essentially related methods: (i) one starts from a quantum simple Lie algebra, makes it affine, thus giving rise to Jimbo's equations $[11,25]$, and then solves them; (ii) one begins with a braid group representation associated with a quantum simple Lie algebra and then tries to

Received 6th April, 1994.

Y.Z. Zhang thanks V.N. Tolstoy for clarifying discussions. The financial support of the Australian Research Council is gratefully acknowledged.

Copyright Clearance Centre, Inc. Serial-fee code: 0004-9729/95 \$A2.00+0.00. 
introduce a spectral parameter in such a way that the spectral-dependent QYBE is satisfied [13]. In practice, it is usually more convenient to use (i) combined with some features of (ii), as shown in $[24,7,5]$. Abelian Yang-Baxterisation, for cases where the decomposition of the tensor product of a representation with itself is multiplicity-free, has been extensively studied by many authors for small representations. The "fusion" method was invented to cope with larger representations, but it is not easy to apply in practice. Very few attempts have been made in the non-Abelian case, where the tensor product decomposition has finite multiplicities, because of the associated complexities. For these reasons, it is highly desirable to develop more effective and general methods of constructing solutions of the spectral-dependent QYBE.

We have presented recently a new way [26] of obtaining spectral-dependent $R$ matrices associated with simple quantum Lie algebras. The idea is essentially to reverse the above process. More precisely, we start from the universal $R$-matrix of a quantum affine algebra $U_{q}\left(\mathcal{G}^{(1)}\right)$ and then evaluate it in the finite-dimensional loop representation $V(z)$ of $U_{q}\left(\mathcal{G}^{(1)}\right)$ which is known to define also the representation $V \otimes \mathbf{C}\left(z, z^{-1}\right)$ of the corresponding simple quantum Lie algebra $U_{q}(\mathcal{G})$. In this way, we introduce a spectral parameter $z$ automatically and obtain a spectral parameter-dependent solution of the QYBE. Our approach is an extension of that initiated by Khoroshkin and Tolstoy [15], who considered the simplest case of the fundamental representation of $U_{q}\left(A_{1}\right)$ which has a classical analogue $[3,21]$. A remarkable advantage of this approach is that it is totally irrelevant whether or not the tensor product decomposition is multiplicity-free, and whether or not the representations being tensored are the same or different.

Moreover, considering different gradations of the quantum affine Lie algebras, our approach leads to quantum $R$-matrices which obey the QYBE but not the intertwiner property and thus cannot be directly obtained by the Yang-Baxterisation and fusion methods. These results will be published elsewhere [6].

The present paper continues the investigation in the authors' previous papers [26, 6] to other interesting cases. It is hoped that the explicit $R$-matrix formulae derived in this paper will turn out to be useful in physical applications.

The paper is structured in the following fashion. In Sections 1 and 2 we give an account of the necessary background. In Section 3, we present the universal $R$-matrix with explicit spectral dependence for $U_{q}\left(A_{1}\right)$ and $U_{q}\left(A_{2}\right)$. In Section 4 , we evaluate the spectral-dependent universal $R$-matrix in some concrete representations and reproduce some well-known results. We also obtain a quite explicit formula for the spectraldependent $R$-matrix on the module $V_{(3)} \times V_{(6)}$ of $U_{q}\left(A_{2}\right)$. Some concluding remarks are made in Section 5, while some technical details are relegated to the Appendix. 


\section{Quantum Affine lie Algebras}

We start with the definition of the non-twisted quantum affine Lie algebra $U_{q}\left(\mathcal{G}^{(1)}\right)$. Let $A^{0}=\left(a_{i j}\right)_{1 \leqslant i, j \leqslant r}$ be a symmetrisable Cartan matrix. Let $\mathcal{G}$ stand for the finitedimensional simple Lie algebra associated with the symmetric Cartan matrix $A_{\mathrm{sym}}^{\mathbf{0}}=$ $\left(a_{i j}^{\text {sym }}\right)=\left(\alpha_{i}, \alpha_{j}\right), i, j=1,2, \ldots, r$, where $r$ is the rank of $\mathcal{G}$. Let $A=\left(a_{i j}\right)_{0 \leqslant i, j \leqslant r}$ be a symmetrisable, generalised Cartan matrix in the sense of Kac [14]. Let $\mathcal{G}^{(1)}$ denote the non-twisted affine Lie algebra associated with the corresponding symmetric Cartan matrix $A_{\text {sym }}=\left(a_{i j}^{\text {sym }}\right)=\left(\alpha_{i}, \alpha_{j}\right), i, j=0,1, \ldots, r$. Then the quantum algebra $U_{q}\left(\mathcal{G}^{(1)}\right)$ is defined to be a Hopf algebra with generators: $\left\{E_{i}, F_{i}, q^{h_{i}}(i=0,1, \ldots, r), q^{d}\right\}$ and relations,

$$
\begin{gathered}
q^{h} \cdot q^{h^{\prime}}=q^{h+h^{\prime}} \quad\left(h, h^{\prime}=h_{i}(i=0,1, \ldots, r), d\right) \\
q^{h} E_{i} q^{-h}=q^{\left(h, \alpha_{i}\right)} E_{i}, q^{h} F_{i} q^{-h}=q^{-\left(h, \alpha_{i}\right)} F_{i} \\
{\left[E_{i}, F_{j}\right]=\delta_{i j} \frac{q^{h_{i}}-q^{-h_{i}}}{q-q^{-1}}} \\
\left(\operatorname{ad}_{q} E_{i}\right)^{1-a_{i j}} E_{j}=0, \quad\left(\operatorname{ad}_{q^{-1}} F_{i}\right)^{1-a_{i j}} F_{j}=0 \quad(i \neq j)
\end{gathered}
$$

where

$$
\left(\operatorname{ad}_{q} x_{\alpha}\right) x_{\beta}=\left[x_{\alpha}, x_{\beta}\right]_{q}=x_{\alpha} x_{\beta}-q^{(\alpha, \beta)} x_{\beta} x_{\alpha} .
$$

The algebra $U_{q}\left(\mathcal{G}^{(1)}\right)$ is a Hopf algebra with coproduct, counit and antipode, much as in the case of $U_{q}(\mathcal{G})$ : explicitly, the coproduct is defined by

$$
\begin{aligned}
& \Delta\left(q^{h}\right)=q^{h} \otimes q^{h}, \quad h=h_{i}, d \\
& \Delta\left(E_{i}\right)=E_{i} \otimes 1+q^{-h_{i}} \otimes E_{i} \\
& \Delta\left(F_{i}\right)=1 \otimes F_{i}+F_{i} \otimes q^{h_{i}}, \quad i=0,1, \ldots, r .
\end{aligned}
$$

Formulae for the co-unit and antipode may also be given, but are not required below.

Let $\Delta^{\prime}$ be the opposite coproduct: $\Delta^{\prime}=T \Delta, T(x \otimes y)=y \otimes x$ for all $x, y \in$ $U_{q}\left(\mathcal{G}^{(1)}\right)$. Then $\Delta$ and $\Delta^{\prime}$ are related by the universal $R$-matrix $R$ in $U_{q}\left(\mathcal{G}^{(1)}\right) \otimes$ $U_{q}\left(\mathcal{G}^{(1)}\right)$ satisfying

$$
\Delta^{\prime}(x) R=R \Delta(x), \quad x \in U_{q}\left(\mathcal{G}^{(1)}\right)
$$

We define an anti-involution $\theta$ on $U_{q}\left(\mathcal{G}^{(1)}\right)$ by

$$
\theta\left(q^{h}\right)=q^{-h}, \theta\left(E_{i}\right)=F_{i}, \theta\left(F_{i}\right)=E_{i}, \theta(q)=q^{-1},
$$


which extends uniquely to an algebra anti-involution on all of $U_{q}\left(\mathcal{G}^{(1)}\right)$ so that $\theta(a b)=$ $\theta(b) \theta(a)$ for all $a, b \in U_{q}\left(\mathcal{G}^{(1)}\right)$. Throughout the paper, we use the notations:

$$
\begin{aligned}
(n)_{q} & =\frac{1-q^{-n}}{1-q^{-1}},[n]_{q}=\frac{q^{n}-q^{-n}}{q-q^{-1}}, q_{\alpha}=q^{(\alpha, \alpha)} \\
\exp _{q}(x) & =\sum_{n \geqslant 0} \frac{x^{n}}{(n)_{q} !},(n)_{q} !=(n)_{q}(n-1)_{q} \ldots(1)_{q} .
\end{aligned}
$$

3. Universal $R$-Matrices for $U_{q}\left(A_{1}^{(1)}\right)$ and $U_{q}\left(A_{2}^{(1)}\right)$

This section is devoted to a brief review of the construction of the universal $R$ matrix for $U_{q}\left(A_{1}^{(1)}\right)$ and $U_{q}\left(A_{2}^{(1)}\right)[16,27,28]$. We start with the rank 2 case. Fix a normal ordering in the positive root system $\Delta_{+}$of $A_{1}^{(1)}$ :

$$
\alpha, \alpha+\delta, \ldots, \alpha+n \delta, \ldots, \delta, 2 \delta, \ldots, m \delta, \ldots, \ldots,(\delta-\alpha)+l \delta, \ldots, \delta-\alpha,
$$

where $\alpha$ and $\delta-\alpha$ are simple roots; $\delta$ is the minimal positive imaginary root. Construct Cartan-Weyl generators $E_{\gamma}, F_{\gamma}=\theta\left(E_{\gamma}\right), \gamma \in \Delta_{+}$of $U_{q}\left(A_{1}^{(1)}\right)$ as follows: define

$$
\begin{aligned}
\widetilde{E_{\delta}} & =[(\alpha, \alpha)]_{q}^{-1}\left[E_{\alpha}, E_{\delta-\alpha}\right]_{q} \\
E_{\alpha+n \delta} & =(-1)^{n}\left(\operatorname{ad} \widetilde{E_{\delta}}\right)^{n} E_{\alpha} \\
E_{(\delta-\alpha)+n \delta} & =\left(\operatorname{ad} \widetilde{E_{\delta}}\right)^{n} E_{\delta-\alpha}, \ldots \\
\widetilde{E}_{n \delta} & =[(\alpha, \alpha)]_{q}^{-1}\left[E_{\alpha+(n-1) \delta}, E_{\delta-\alpha}\right]_{q}
\end{aligned}
$$

(i) for any $n>0$, there exists a unique element $E_{n \delta}[16]$ satisfying $\left[E_{n \delta}, E_{m \delta}\right]=0$ for any $n, m>0$ and the relation

$$
\widetilde{E}_{n \delta}=\sum_{p_{1}+2 p_{2}+\cdots+n p_{n}=n} \frac{\left(q^{(\alpha, \alpha)}-q^{-(\alpha, \alpha)}\right)^{\sum_{i} p_{i}-1}}{p_{1} ! \ldots p_{n} !} E_{\delta}^{p_{1}} E_{2 \delta}^{p_{2}} \cdots E_{n \delta}^{p_{n}} .
$$

(ii) the vectors $E_{\gamma}$ and $F_{\gamma}=\theta\left(E_{\gamma}\right), \gamma \in \Delta_{+}$are the Cartan-Weyl generators for $U_{q}\left(A^{(1)}\right)$. The universal $R$-matrix for $U_{q}\left(A_{1}^{(1)}\right)$ may be written as [16]

$$
\begin{aligned}
R= & \left(\prod_{n \geqslant 0} \exp _{q_{\alpha}}\left(\left(q-q^{-1}\right)\left(E_{\alpha+n \delta} \otimes F_{\alpha+n \delta}\right)\right)\right) \cdot \exp \left(\sum_{n>0} n[n]_{q_{\alpha}}^{-1}\left(q_{\alpha}-q_{\alpha}^{-1}\right)\left(E_{n \delta} \otimes F_{n \delta}\right)\right) \\
& \cdot\left(\prod_{n \geqslant 0} \exp _{q_{\alpha}}\left(\left(q-q^{-1}\right)\left(E_{(\delta-\alpha)+n \delta} \otimes F_{(\delta-\alpha)+n \delta}\right)\right)\right) \cdot q^{1 / 2} h_{\alpha} \otimes h_{\alpha}+c \otimes d+d \otimes c
\end{aligned}
$$


where $c=h_{\alpha}+h_{\delta-\alpha}$. The order in the product (10) concides with the chosen normal order (7).

Now consider the rank 3 case. Let $A_{\text {sym }}^{0}=\left(a_{i j}^{\text {sym }}\right), i, j=1,2$ and $\Delta_{+}^{0}$ respectively be the symmetrical Cartan matrix and positive root system of the rank 2 finitedimensional simple Lie algebra $A_{2}$. In what follows we use $A_{\mathrm{sym}}^{0}$ in the form

$$
A_{\mathrm{sym}}^{0}=\left(a_{i j}^{\mathrm{sym}}\right)=\left(\begin{array}{ll}
(\alpha, \alpha) & (\alpha, \beta) \\
(\beta, \alpha) & (\beta, \beta)
\end{array}\right)=\left(\begin{array}{cc}
2 & -1 \\
-1 & 2
\end{array}\right)
$$

The simple roots are $\alpha, \beta$ and $\delta-\psi$ with $\psi=\alpha+\beta$ the highest root of $A_{2}$ :

One fixes the following order in $\Delta_{+}$of $A_{2}^{(1)}$ :

$$
\alpha, \alpha+\beta, \alpha+\delta, \alpha+\beta+\delta, \ldots, \ldots, \alpha+m_{1} \delta, \alpha+\beta+m_{1} \delta, \ldots, \ldots,
$$

$\beta, \beta+\delta, \ldots, \beta+m_{2} \delta, \ldots, \delta, 2 \delta, \ldots, k \delta, \ldots, \ldots,(\delta-\beta)+l_{1} \delta, \ldots, \delta-\beta, \ldots$,

$$
\ldots,(\delta-\alpha)+l_{2} \delta,(\delta-\alpha-\beta)+l_{2} \delta, \ldots, \ldots, \delta-\alpha, \delta-\alpha-\beta \text {, }
$$

where $m_{i}, k, l_{i} \geqslant 0, i=1,2$. We set

$$
\begin{aligned}
& E_{\alpha+\beta}=\left[E_{\alpha}, E_{\beta}\right]_{q}, \quad E_{\delta-\alpha}=\left[E_{\beta}, E_{\delta-\alpha-\beta}\right]_{q} \\
& E_{\delta-\beta}=\left[E_{\alpha}, E_{\delta-\alpha-\beta}\right]_{q}
\end{aligned}
$$

and use the formula for $E_{\gamma+n \delta}$ and $E_{(\delta-\gamma)+n \delta}, \gamma \in \Delta_{+}^{0}$, given by

$$
\begin{aligned}
\widetilde{E}_{\delta}^{(i)} & =\left[\left(\alpha_{i}, \alpha_{i}\right)\right]_{q}^{-1}\left[E_{\alpha_{i}}, E_{\delta-\alpha_{i}}\right]_{q}, \quad \alpha_{i}=\alpha, \beta, \alpha+\beta \\
E_{\alpha_{i}+n \delta} & =(-1)^{n}\left(\operatorname{ad} \tilde{E}_{\delta}^{(i)}\right)^{n} E_{\alpha_{i}} \\
E_{\delta-\alpha_{i}+n \delta} & =\left(\operatorname{ad} \tilde{E}_{\delta}^{(i)}\right)^{n} E_{\delta-\alpha_{i}}, \ldots \\
\widetilde{E}_{n \delta}^{(i)} & =\left[\left(\alpha_{i}, \alpha_{i}\right)\right]_{q}^{-1}\left[E_{\alpha_{i}+(n-1) \delta}, E_{\delta-\alpha_{i}}\right]_{q},
\end{aligned}
$$

where $\left[\widetilde{E}_{n \delta}^{(i)}, \widetilde{E}_{m \delta}^{(j)}\right]=0$ for any $n, m>0$. One has the following statment similar to the case of $U_{q}\left(A_{1}^{(1)}\right)$ :

(i) there exists a unique element $E_{n \delta}^{(i)}, n>0$ satisfying $\left[E_{n \delta}^{(i)}, E_{m \delta}^{(j)}\right]=0$ for any $n, m>0$ and the relation $\left(\alpha_{i}=\alpha, \beta\right)$

$$
\tilde{E}_{n \delta}^{(i)}=\sum_{p_{1}+2 p_{2}+\cdots+n p_{n}} \frac{\left(q^{\left(\alpha_{i}, \alpha_{i}\right)}-q^{-\left(\alpha_{i}, \alpha_{i}\right)}\right)^{\sum_{i} p_{i}-1}}{p_{1} ! \cdots p_{n} !}\left(E_{\delta}^{(i)}\right)^{p_{1}}\left(E_{2 \delta}^{(i)}\right)^{p_{2}} \cdots\left(E_{n \delta}^{(i)}\right)^{p_{n}}
$$

(ii) the vectors $E_{\gamma}$ and $F_{\gamma}=\theta\left(E_{\gamma}\right), \gamma \in \Delta_{+}$are the Cartan-Weyl generators for $U_{q}\left(A_{2}^{(1)}\right)$. 
One can show $[16,27]$ (see, in particular, [27]) that the universal $R$-matrix for $U_{q}\left(A_{2}^{(1)}\right)$ takes the explicit form

$$
\begin{aligned}
& R=\prod_{\gamma<\delta} \exp _{q_{\gamma}}\left(\left(q-q^{-1}\right)\left(E_{\gamma} \otimes F_{\gamma}\right)\right) \\
& \cdot \exp \left(\sum_{n>0} \sum_{i, j=1}^{2} C_{i j}^{n}(q)\left(q-q^{-1}\right)\left(E_{n \delta}^{(i)} \otimes F_{n \delta}^{(j)}\right)\right) \\
& \cdot \prod_{\gamma>\delta} \exp _{q_{\gamma}}\left(\left(q-q^{-1}\right)\left(E_{\gamma} \otimes F_{\gamma}\right)\right) \\
& \cdot \sum^{\sum_{i, j=1}^{2}}\left(\begin{array}{c}
\left.a_{n \mathrm{ym}}^{-1}\right)^{i j} h_{i} \otimes h_{j}+c \otimes d+d \otimes c
\end{array}\right.
\end{aligned}
$$

where $c=h_{0}+h_{\psi}$, the order in the product of (16) is defined by (12), and the constants $C_{i j}^{n}(q)$ are given by

$$
\left(C_{i j}^{n}(q)\right)=\left(C_{j i}^{n}(q)\right)=\frac{n}{[n]_{q}} \frac{[2]_{q}^{2}}{q^{2 n}+1+q^{-2 n}}\left(\begin{array}{cc}
q^{n}+q^{-n} & (-1)^{n} \\
(-1)^{n} & q^{n}+q^{-n}
\end{array}\right) .
$$

\section{Universal $R$-Matrices with Spectral Parameters}

We turn now to the main object of the paper. We shall determine explicitly spectral-dependent universal $R$-matrices for $U_{q}\left(A_{1}\right)$ and $U_{q}\left(A_{2}\right)$ by using the universal $R$-matrices (10) and (16) for $U_{q}\left(A_{1}^{(1)}\right)$ and $U_{q}\left(A_{2}^{(1)}\right)$, respectively.

From the Appendix it follows that for any $z \in \mathbf{C}^{\times}$, there is a homomorphism of algebras $\operatorname{ev}_{z}: U_{q}\left(A_{1}^{(1)}\right) \rightarrow U_{q}\left(A_{1}\right)$ given by

$$
\begin{aligned}
& \operatorname{ev}_{z}\left(E_{\alpha}\right)=E_{\alpha}, \quad \operatorname{ev}_{z}\left(F_{\alpha}\right)=F_{\alpha}, \quad \operatorname{ev}_{z}\left(h_{\alpha}\right)=h_{\alpha}, \quad \operatorname{ev} z(c)=0 \\
& \operatorname{ev}_{z}\left(E_{\delta-\alpha}\right)=z F_{\alpha}, \quad \operatorname{ev}_{z}\left(F_{\delta-\alpha}\right)=z^{-1} E_{\alpha}, \quad \operatorname{ev}_{z}\left(h_{\delta-\alpha}\right)=-h_{\alpha} .
\end{aligned}
$$

Then suppressing " $\mathrm{ev}_{z}$ " for notational convenience, and through straightforward calculations and induction in $n$, using (18) and the defining relations (8), we obtain

$$
\begin{aligned}
E_{\alpha+n \delta} & =(-1)^{n} z^{n} q^{-n h_{\alpha}} E_{\alpha} \\
F_{\alpha+n \delta} & =(-1)^{n} z^{-n} F_{\alpha} q^{n h_{\alpha}} \\
E_{(\delta-\alpha)+n \delta} & =(-1)^{n} z^{n+1} F_{\alpha} q^{-n h_{\alpha}} \\
F_{(\delta-\alpha)+n \delta} & =(-1)^{n} z^{-n-1} q^{n h_{\alpha}} E_{\alpha} \\
\tilde{E}_{n \delta} & =[2]_{q}^{-1}(-1)^{n-1} z^{n} q^{-(n-1) h_{\alpha}}\left(E_{\alpha} F_{\alpha}-q^{-2 n} F_{\alpha} E_{\alpha}\right) \\
\tilde{F}_{n \delta} & =[2]_{q}^{-1}(-1)^{n-1} z^{-n} q^{(n-1) h_{\alpha}}\left(F_{\alpha} E_{\alpha}-q^{2 n} E_{\alpha} F_{\alpha}\right) .
\end{aligned}
$$


We introduce the primed and $z$-independent quantities, $\widetilde{E}_{n \delta}^{\prime}$ and $\widetilde{F}_{n \delta}^{\prime}$ which are obtained from the corresponding unprimed quantities in (19) by removing the parameter $z$, and determine $E_{n \delta}^{\prime}$ and $F_{n \delta}^{\prime}$ using the following equalities of formal power series:

$$
\begin{aligned}
1+\left(q_{\alpha}-q_{\alpha}^{-1}\right) \sum_{k=1}^{\infty} \widetilde{E}_{k \delta}^{\prime} u^{k} & =\exp \left(\left(q_{\alpha}-q_{\alpha}^{-1}\right) \sum_{l=1}^{\infty} E_{l \delta}^{\prime} u^{l}\right) \\
1-\left(q_{\alpha}-q_{\alpha}^{-1}\right) \sum_{k=1}^{\infty} \widetilde{F}_{k \delta}^{\prime} u^{-k} & =\exp \left(-\left(q_{\alpha}-q_{\alpha}^{-1}\right) \sum_{l=1}^{\infty} F_{l \delta}^{\prime} u^{-1}\right)
\end{aligned}
$$

which are variants of (9). Then from (10) we deduce the universal $R$-matrix of $U_{q}\left(A_{1}\right)$ with the explicit dependence of spectral parameter, $R(x, y) \equiv\left(\mathrm{ev}_{x} \otimes \mathrm{ev}_{y}\right) R$,

$$
\begin{aligned}
R(x, y)=\prod_{n \geqslant 0} \exp _{q_{\alpha}}\left(\left(q-q^{-1}\right)\left(\frac{x}{y}\right)^{n}\left(q^{-n h_{\alpha}} E_{\alpha} \otimes F_{\alpha} q^{n h_{\alpha}}\right)\right) \\
\cdot \exp \left(\sum_{n>0} n[n]_{q_{\alpha}}^{-1}\left(q_{\alpha}-q_{\alpha}^{-1}\right)\left(\frac{x}{y}\right)^{n}\left(E_{n \delta}^{\prime} \otimes F_{n \delta}^{\prime}\right)\right) \\
\quad \cdot \prod_{n \geqslant 0} \exp _{q_{\alpha}}\left(\left(q-q^{-1}\right)\left(\frac{x}{y}\right)^{n+1}\left(F_{\alpha} q^{-n h_{\alpha}} \otimes q^{n h_{\alpha}} E_{\alpha}\right)\right) \cdot q^{\left(h_{\alpha} \otimes h_{\alpha}\right) / 2} .
\end{aligned}
$$

We now consider the case of $U_{q}\left(A_{2}^{(1)}\right)$. We have (see Appendix) that for any $z \in \mathbf{C}^{\times}$, there is a homomorphism of algebras $\mathrm{ev}_{z}: U_{q}\left(A_{2}^{(1)}\right) \rightarrow U_{q}\left(A_{2}\right)$ given by

$$
\begin{aligned}
\mathrm{ev}_{z}\left(E_{\alpha}\right) & =E_{\alpha}, \quad \mathrm{ev}_{z}\left(F_{\alpha}\right)=F_{\alpha}, \quad \mathrm{ev}_{z}\left(h_{\alpha}\right)=h_{\alpha} \\
\mathrm{ev}_{z}\left(E_{\beta}\right) & =E_{\beta}, \quad \mathrm{ev}_{z}\left(F_{\beta}\right)=F_{\beta}, \quad \mathrm{ev}_{z}\left(h_{\beta}\right)=h_{\beta} \\
\mathrm{ev}_{z}\left(E_{\delta-\alpha-\beta}\right) & =z F_{\alpha+\beta} q^{\left(h_{\beta}-h_{\alpha}\right) / 3}, \quad \mathrm{ev}_{z}\left(F_{\delta-\alpha-\beta}\right)=z^{-1} q^{\left(h_{\alpha}-h_{\beta}\right) / 3} E_{\alpha+\beta} \\
\mathrm{ev}_{z}\left(h_{\delta-\alpha-\beta}\right) & =-h_{\alpha+\beta}, \quad \mathrm{ev}_{z}(c)=0
\end{aligned}
$$

Again suppressing " $\mathrm{ev}_{z}$ " and using (22) and the defining relations of the generators, equations (14) and (15), we obtain

$$
\begin{aligned}
E_{\alpha+n \delta} & =(-1)^{n} z^{n} q^{-n h_{\alpha}} E_{\alpha} q^{-n\left(h_{\alpha}+2 h_{\beta}\right) / 3} \\
F_{\alpha+n \delta} & =(-1)^{n} z^{-n} q^{n\left(h_{\alpha}+2 h_{\beta}\right) / 3} F_{\alpha} q^{n h_{\alpha}} \\
E_{\alpha+\beta+n \delta} & =(-1)^{n} z^{n} q^{-n h_{\alpha+\beta}} E_{\alpha+\beta} q^{n\left(h_{\beta}-h_{\alpha}\right) / 3} \\
F_{\alpha+\beta+n \delta} & =(-1)^{n} z^{-n} q^{n\left(h_{\alpha}-h_{\beta}\right) / 3} F_{\alpha+\beta} q^{n h_{\alpha+\beta}} \\
E_{\beta+n \delta} & =(-1)^{n}[2]_{q}^{-n} z^{n} q^{n}\left\{\left(\operatorname{ad}_{q^{\prime}-1} \mathcal{E}\right)^{n} E_{\beta}\right\} q^{n\left(h_{\beta}-h_{\alpha}\right) / 3}
\end{aligned}
$$




$$
\begin{aligned}
& F_{\beta+n \delta}=[2]_{q}^{-n} z^{-n} q^{n\left(h_{\alpha}-h_{\beta}\right) / 3}\left(\operatorname{ad}_{q^{-1}} \mathcal{F}\right)^{n} F_{\beta} \\
& E_{(\delta-\beta)+n \delta}=[2]_{q}^{-n} z^{n+1} q^{-n}\left\{\left(\operatorname{ad}_{q}^{\prime} \mathcal{E}\right)^{n}\left(\operatorname{ad}_{q^{-2}} E_{\alpha}\right) F_{\alpha+\beta}\right\} q^{(n+1)\left(h_{\beta}-h_{\alpha}\right) / 3} \\
& F_{(\delta-\beta)+n \delta}=(-1)^{n}[2]_{q}^{-n} z^{-n-1} q^{(n+1)\left(h_{\alpha}-h_{\beta}\right) / \mathrm{s}}\left(\operatorname{ad}_{q}^{\prime} \mathcal{F}\right)^{n}\left(\operatorname{ad}_{q^{2}} E_{\alpha+\beta}\right) F_{\alpha} \\
& E_{(\delta-\alpha)+n \delta}=(-1)^{n} z^{n+1} q^{-(n+1)\left(h_{\alpha}+2 h_{\beta}\right) / 3} F_{\alpha} q^{-n h_{\alpha}} \\
& F_{(\delta-\alpha)+n \delta}=(-1)^{n} z^{-n-1} q^{n h_{\alpha}} E_{\alpha} q^{(n+1)\left(h_{\alpha}+2 h_{\beta}\right) / 3} \\
& E_{(\delta-\alpha-\beta)+n \delta}=(-1)^{n} z^{n+1} q^{(n+1)\left(h_{\beta}-h_{\alpha}\right) / 3} F_{\alpha+\beta} q^{-n h_{\alpha+\beta}} \\
& F_{(\delta-\alpha-\beta)+n \delta}=(-1)^{n} z^{-n-1} q^{n h_{\alpha+\beta}} E_{\alpha+\beta} q^{(n+1)\left(h_{\alpha}-h_{\beta}\right) / 3} \\
& \widetilde{E}_{n \delta}^{(\alpha)}=(-1)^{n-1}[2]_{q}^{-1} z^{n}\left(E_{\alpha} F_{\alpha}-q^{-2 n} F_{\alpha} E_{\alpha}\right) q^{-(n-1) h_{\alpha}} q^{-n\left(h_{\alpha}+2 h_{\beta}\right) / 3} \\
& \tilde{F}_{n \delta}^{(\alpha)}=(-1)^{n-1}[2]_{q}^{-1} z^{-n} q^{(n-1) h_{\alpha}} q^{n\left(h_{\alpha}+2 h_{\beta}\right) / 3}\left(F_{\alpha} E_{\alpha}-q^{2 n} E_{\alpha} F_{\alpha}\right) \\
& \tilde{E}_{n \delta}^{(\beta)}=(-1)^{n}[2]_{q}^{-n} z^{n} q^{n-2}\left\{\left(\operatorname{ad}_{q^{-n+2}}^{\prime} \mathcal{F}^{\prime}\right) \cdot\left(\operatorname{ad}_{q^{-1}}^{\prime} \mathcal{E}\right)^{n-1} E_{\beta}\right\} q^{n\left(h_{\beta}-h_{\alpha}\right) / 3} \\
& \widetilde{F}_{n \delta}^{(\beta)}=[2]_{q}^{-n} z^{-n} q^{n-1} q^{n\left(h_{\alpha}-h_{\beta}\right) / 3}\left(\operatorname{ad}_{q^{-n+2}}^{\prime} \mathcal{E}^{\prime}\right) \cdot\left(\operatorname{ad}_{q^{-1}}^{\prime} \mathcal{F}\right)^{n-1} F_{\beta},
\end{aligned}
$$

where $\left(\operatorname{ad}_{Q}^{\prime} \mathcal{A}\right) \cdot \mathcal{B} \equiv \mathcal{A B}-Q \mathcal{B} \mathcal{A}$, and

$$
\begin{aligned}
\mathcal{E} & =\left(\operatorname{ad}_{q^{-1}}^{\prime} E_{\beta}\right)\left(\operatorname{ad}_{q^{-2}}^{\prime} E_{\alpha}\right) F_{\alpha+\beta}, \quad \mathcal{F}=\left(\operatorname{ad}_{q}^{\prime}\left(\operatorname{ad}_{q^{2}}^{\prime} E_{\alpha+\beta}\right) F_{\alpha}\right) F_{\beta} \\
\mathcal{E}^{\prime} & =E_{\alpha+\beta} F_{\alpha}-q^{2} F_{\alpha} E_{\alpha+\beta}, \quad \mathcal{F}^{\prime}=E_{\alpha} F_{\alpha+\beta}-q^{-2} F_{\alpha+\beta} E_{\alpha} .
\end{aligned}
$$

We introduce the primed and $z$-independent quantities $\widetilde{E}_{n \delta}^{\prime(\alpha)}, \widetilde{F}_{n \delta}^{\prime(\alpha)}, \tilde{E}_{n \delta}^{\prime(\beta)}, \tilde{F}_{n \delta}^{\prime(\beta)}$, $E_{\beta+n \delta}^{\prime}, F_{\beta+n \delta}^{\prime} E_{(\delta-\beta)+n \delta}^{\prime}$ and $F_{(\delta-\beta)+n \delta}^{\prime}$, which are obtained from the corresponding unprimed quantities in (23) by suppressing the parameter $z$, and determine $E_{n \delta}^{\prime(i)}, F_{n \delta}^{\prime(i)}$ by the following equalities of formal series: $\left(\alpha_{i}=\alpha, \beta\right)$

$$
\begin{gathered}
k \delta u^{k}=\exp \left(\left(q_{\alpha_{i}}-q_{\alpha_{i}}^{-1}\right) \sum_{l=1}^{\infty} E_{l \delta}^{\prime(i)} u^{l}\right) \\
1-\left(q_{\alpha_{i}}-q_{\alpha_{i}}^{-1}\right) \sum_{k=1}^{\infty} \tilde{F}_{k \delta}^{\prime(i)} u^{-k}=\exp \left(-\left(q_{\alpha_{i}}-q_{\alpha_{i}}^{-1}\right) \sum_{l=1}^{\infty} F_{l \delta}^{\prime(i)} u^{-l}\right)
\end{gathered}
$$

which are variants of (15). Then from (16) we may deduce the universal $R$-matrix of $U_{q}\left(A_{2}\right)$ with the explicit dependence on a spectral parameter, $R(x, y) \equiv\left(\operatorname{ev}_{x} \otimes e_{y}\right) R$, given by

$$
\begin{aligned}
R(x, y)=\prod_{n \geqslant 0} & \left\{\operatorname { e x p } _ { q _ { \alpha } } \left(( q - q ^ { - 1 } ) ( \frac { x } { y } ) ^ { n } \left(q^{-n h_{\alpha}} E_{\alpha} q^{-n\left(h_{\alpha}+2 h_{\beta}\right) / 3}\right.\right.\right. \\
\otimes & \left.\left.q^{n\left(h_{\alpha}+2 \beta\right) / 3} F_{\alpha} q^{n h_{\alpha}}\right)\right)
\end{aligned}
$$




$$
\begin{aligned}
& \cdot \exp _{q_{\alpha+\beta}}\left(( q - q ^ { - 1 } ) ( \frac { x } { y } ) ^ { n } \left(q^{-n h_{\alpha+\beta}} E_{\alpha+\beta} q^{n\left(h_{\beta}-h_{\alpha}\right) / s}\right.\right. \\
& \left.\left.\left.\otimes q^{n\left(h_{\alpha}-\beta\right) / 3} F_{\alpha+\beta} q^{n h_{\alpha+\beta}}\right)\right)\right\} \\
& \cdot \prod_{n \geqslant 0} \exp _{q_{\beta}}\left(\left(q-q^{-1}\right)\left(\frac{x}{y}\right)^{n}\left(E_{\beta+n \delta}^{\prime} \otimes F_{\beta+n \delta}^{\prime}\right)\right) \\
& \cdot \exp \left(\sum_{n>0} \sum_{i, j=1}^{2} C_{i j}^{n}(q)\left(q-q^{-1}\right)\left(\frac{x}{y}\right)^{n}\left(E_{n \delta}^{\prime(i)} \otimes F_{n \delta}^{\prime(j)}\right)\right) \\
& \cdot \prod_{n \geqslant 0} \exp _{q_{\beta}}\left(\left(q-q^{-1}\right)\left(\frac{x}{y}\right)^{n+1}\left(E_{(\delta-\beta)+n \delta}^{\prime} \otimes F_{(\delta-\beta)+n \delta}^{\prime}\right)\right) \\
& \cdot \prod_{n \geqslant 0}\left\{\operatorname { e x p } _ { q _ { \alpha } } \left(( q - q ^ { - 1 } ) ( \frac { x } { y } ) ^ { n + 1 } \left(q^{-(n+1)\left(h_{\alpha}+2 h_{\beta}\right) / 3} F_{\alpha} q^{-n h_{\alpha}}\right.\right.\right. \\
& \left.\left.\otimes q^{n h_{\alpha}} E_{\alpha} q^{(n+1)\left(h_{\alpha}+2 h_{\beta}\right) / 3}\right)\right) \\
& \cdot \exp _{q_{\alpha+\beta}}\left(( q - q ^ { - 1 } ) ( \frac { x } { y } ) ^ { n + 1 } \left(q^{(n+1)\left(h_{\beta}-h_{\alpha}\right) / 3} F_{\alpha+\beta} q^{-n h_{\alpha+\beta}}\right.\right. \\
& \left.\left.\left.\otimes q^{n h_{\alpha+\beta}} E_{\alpha+\beta} q^{(n+1)\left(h_{\alpha}-h_{\beta}\right) / 3}\right)\right)\right\} \cdot q^{\sum_{i, j=1}^{2}\left(a_{s y m}^{-1}\right)^{i j} h_{i} \otimes h_{j}} .
\end{aligned}
$$

\section{Applications}

To illustrate the general theory developed in the previous section, we present a detailed study of the spectral-dependent $R$-matrix for some concrete and interesting representations.

First consider the $U_{q}\left(A_{1}^{(1)}\right)$ case. Let $V_{l / 2}, l \in \mathbf{Z}_{+}$denote the $(l+1)$-dimensional module of $U_{q}\left(A_{1}\right)$ (spin $l / 2$ representation) with basis $\left\{v_{m}^{(l)} \mid 0 \leqslant m \leqslant l\right\}$. We have for this representation,

$$
\begin{aligned}
h_{\alpha} v_{m}^{(l)} & =(l-2 m) v_{m}^{(l)} \\
E_{\alpha} v_{m}^{(l)} & =[l-m+1]_{q} v_{m-1}^{(l)} \\
F_{\alpha} v_{m}^{(l)} & =[m+1]_{q} v_{m+1}^{(l)} \\
E_{n \delta}^{\prime} v_{m}^{(l)} & =[2]_{q}^{-1} \frac{(-1)^{n-1}}{n} q^{n m}\left([n(l-m)]_{q}-q^{-n(l+2)}[n m]_{q}\right) v_{m}^{(l)} \\
F_{n \delta}^{\prime} v_{m}^{(l)} & =[2]_{q}^{-1} \frac{(-1)^{n-1}}{n} q^{-n m}\left([n(l-m)]_{q}-q^{n(l+2)}[n m]_{q}\right) v_{m}^{(l)}
\end{aligned}
$$

where it is understood that $v_{m}^{(l)}$ is identically zero if $m>l$ or $m<0$. These results 
are obtained by straightforward computation plus induction in $n$.

(i) for the spin $1 / 2$ representation, we have from (26)

$$
h_{\alpha}=\left(\begin{array}{cc}
1 & 0 \\
0 & -1
\end{array}\right), \quad E_{\alpha}\left(\begin{array}{ll}
0 & 1 \\
0 & 0
\end{array}\right), \quad F_{\alpha}=\left(\begin{array}{ll}
0 & 0 \\
1 & 0
\end{array}\right)
$$

(27)

$$
E_{n \delta}^{\prime}=[2]_{q}^{-1} \frac{(-1)^{n-1}}{n}[n]_{q}\left(\begin{array}{cc}
1 & 0 \\
0 & -q^{-2 n}
\end{array}\right), \quad F_{n \delta}^{\prime}=[2]_{q}^{-1} \frac{(-1)^{n-1}}{n}[n]_{q}\left(\begin{array}{cc}
1 & 0 \\
0 & -q^{2 n}
\end{array}\right)
$$

We apply (21) to $V_{1 / 2} \otimes V_{1 / 2}$, where $V_{1 / 2}$ carries the spin- $1 / 2$ representation of $U_{q}\left(A_{1}\right)$. Using (27), it follows from (21) that

$$
R_{1 / 2,1 / 2}(x, y)=f_{q}(x, y) \cdot\left(\begin{array}{llll}
1 & & & \\
& \frac{q^{-1}(y-x)}{y-q^{-2} x} & \frac{y\left(1-q^{-2}\right)}{y-q^{-2} x} & \\
& \frac{x\left(1-q^{-2}\right)}{y-q^{-2} x} & \frac{q^{-1}(y-x)}{y-q^{-2} x} & \\
& & & 1
\end{array}\right)
$$

where

$$
f_{q}(x, y)=q^{1 / 2} \cdot \exp \left(\sum_{n>0} \frac{q^{n}-q^{-n}}{q^{n}+q^{-n}} \frac{(x / y)^{n}}{n}\right)
$$

and use has been made of the notation:

$$
(A \otimes B)=\left(\begin{array}{cccc}
A_{11} B & A_{12} B & \cdots & A_{1 N} B \\
\vdots & \vdots & \ddots & \vdots \\
A_{M 1} B & A_{M 2} B & \cdots & A_{M N} B
\end{array}\right)
$$

Equation (28) reproduces a well-known result [12], up to the scalar factor $f_{q}(x, y)$. Khoroshkin and Tolstoy [16] obtained (29) directly from (10).

(ii) for the spin 1 representation, (26) gives

$$
h_{\alpha}=\left(\begin{array}{ccc}
2 & 0 & 0 \\
0 & 0 & 0 \\
0 & 0 & -2
\end{array}\right), \quad E_{\alpha}=\left(\begin{array}{ccc}
0 & {[2]_{q}} & 0 \\
0 & 0 & 1 \\
0 & 0 & 0
\end{array}\right), \quad F_{\alpha}=\left(\begin{array}{ccc}
0 & 0 & 0 \\
1 & 0 & 0 \\
0 & {[2]_{q}} & 0
\end{array}\right)
$$

(31) $F_{n \delta}^{\prime}=[2]_{q}^{-1} \frac{(-1)^{n-1}}{n}[n]_{q}\left(\begin{array}{ccc}q^{n}+q^{-n} & 0 & 0 \\ 0 & -q^{n}\left(q^{2 n}-q^{-2 n}\right) & 0 \\ 0 & 0 & -q^{2 n}\left(q^{n}+q^{-n}\right)\end{array}\right)$. 
We now apply (21) to $V_{1 / 2} \otimes V_{1}$ with $V_{1}$ carrying the spin-1 representation of $U_{q}\left(A_{1}\right)$. Using (31), we obtain from (21)

$$
\begin{gathered}
R_{1 / 2,1}(x, y)=\frac{q^{2}\left(y-q^{-1} x\right)}{y-q x} \cdot\left(e_{11}+e_{66}+\frac{q^{-2}(y-q x)}{y-q^{-3} x}\left(e_{33}+e_{44}\right)+\right. \\
\left.+\frac{y q^{-1}\left(1-q^{-2}\right)}{y-q^{-3} x} e_{24}+\frac{x q^{-1}\left(1-q^{-2}\right)}{y-q^{-3} x} e_{53}\right)
\end{gathered}
$$

where (and below) $e_{i j}$ is the matrix satisfying $\left(e_{i j}\right)_{k l}=\delta_{i k} \delta_{j l}$ and $e_{i j} e_{k l}=\delta_{j k} e_{i l}$.

We now turn to the $U_{q}\left(A_{2}^{(1)}\right)$ case. Hence we find that the explicit form of the generators on the fundamental representation of $U_{q}\left(A_{2}\right)$ is given by

$$
\begin{gathered}
h_{\alpha}=\operatorname{diag}(1,-1,0), \quad h_{\beta}=\operatorname{diag}(0,1,-1) \\
E_{\alpha}=\left(\begin{array}{lll}
0 & 1 & 0 \\
0 & 0 & 0 \\
0 & 0 & 0
\end{array}\right), \quad F_{\alpha}=\left(\begin{array}{lll}
0 & 0 & 0 \\
1 & 0 & 0 \\
0 & 0 & 0
\end{array}\right) \\
E_{\alpha+\beta}=\left(\begin{array}{lll}
0 & 0 & 1 \\
0 & 0 & 0 \\
0 & 0 & 0
\end{array}\right), \quad F_{\alpha+\beta}=\left(\begin{array}{lll}
0 & 0 & 0 \\
0 & 0 & 0 \\
1 & 0 & 0
\end{array}\right) \\
E_{\beta+n \delta}^{\prime}=q^{-2 n-n / 3}\left(\begin{array}{ccc}
0 & 0 & 0 \\
0 & 0 & 1 \\
0 & 0 & 0
\end{array}\right), \quad F_{\beta+n \delta}^{\prime}=q^{2 n+n / 3}\left(\begin{array}{lll}
0 & 0 & 0 \\
0 & 0 & 0 \\
0 & 1 & 0
\end{array}\right) \\
E_{(\delta-\beta)+n \delta}^{\prime}=-q^{-2 n-1-(n+1) / 3}\left(\begin{array}{ccc}
0 & 0 & 0 \\
0 & 0 & 0 \\
0 & 1 & 0
\end{array}\right) \\
F_{(\delta-\beta)+n \delta}^{\prime}=-q^{2 n+1+(n+1) / 3}\left(\begin{array}{ccc}
0 & 0 & 0 \\
0 & 0 & 1 \\
0 & 0 & 0
\end{array}\right) \\
E_{n \delta}^{\prime(\alpha)}=[2]_{q}^{-1}(-1)^{n-1} \frac{[n]_{q}}{n} q^{-n / 3} \operatorname{diag}\left(1,-q^{-2 n}, 0\right) \\
F_{n \delta}^{\prime(\alpha)}=[2]_{q}^{-1}(-1)^{n-1} \frac{[n]_{q}}{n} q^{n / 3} \operatorname{diag}\left(1,-q^{2 n}, 0\right) \\
E_{n \delta}^{\prime(\beta)}=[2]_{q}^{-1} \frac{[n]_{q}}{n} q^{-n-n / 3} \operatorname{diag}\left(0,-1, q^{-2 n}\right) \\
F_{n \delta}^{(\beta)}=[2]_{q}^{-1} \frac{[n]_{q}}{n} q^{n+n / 3} \operatorname{diag}\left(0,-1, q^{2 n}\right) .
\end{gathered}
$$

We apply the results in Section 4 to derive the spectral-dependent $R$-matrix associated with $V_{(3)} \otimes V_{(3)}$, where $V_{(3)}$ stands for the fundamental representation of $U_{q}\left(A_{2}\right)$. 
Using (33) we get

$$
\begin{aligned}
& R_{(3),(3)}(x, y)=f_{q}(x, y) \cdot\left(e_{11}+e_{55}+e_{99}+\frac{q^{-1}(y-x)}{y-q^{-2} x}\left(e_{22}+e_{33}+e_{44}+e_{66}+e_{77}+e_{88}\right)\right. \\
& \left.(34) \quad+\frac{y\left(1-q^{-2}\right)}{y-q^{-2} x}\left(e_{24}+e_{37}+e_{68}\right)+\frac{x\left(1-q^{-2}\right)}{y-q^{-2} x}\left(e_{42}+e_{73}+e_{86}\right)\right),
\end{aligned}
$$

where

$$
f_{q}(x, y)=q^{2 / 3} \cdot \exp \left(\sum_{n>0} \frac{q^{2 n}-q^{-2 n}}{q^{2 n}+1+q^{-2 n}} \frac{(x / y)^{n}}{n}\right) .
$$

Again this reproduces a well-known result [12], up to a scalar factor $f_{q}(x, y)$.

We now consider a very interesting case: to extract the spectral depedendent $R$ matrix associated with the module $V_{(3)} \otimes V_{(6)}$ of $U_{q}\left(A_{2}\right)$. As indicated in the introduction, this case could not be treated by means of the usual Yang-Baxterisation procedures, simply because the representations being tensored are different.

We introduce the so-called Gelfand-Tsetlin basis vector $|(m)\rangle$ given by

$$
|(m)\rangle=\left|\left(\begin{array}{ccc}
m_{13} & m_{23} & m_{33} \\
m_{12} & m_{22} \\
& m_{11}
\end{array}\right)\right\rangle .
$$

It can be shown that the action of the generators on these basis vectors is

$$
\begin{aligned}
& h_{\alpha}|(m)\rangle=\left(2 m_{11}-m_{12}-m_{22}\right)|(m)\rangle \\
& h_{\beta}|(m)\rangle=\left(2 m_{12}+2 m_{22}-m_{11}-m_{13}-m_{23}-m_{33}\right)|(m)\rangle \\
& F_{\alpha}\left|\left(\begin{array}{ccc}
m_{13} & m_{23} & m_{33} \\
m_{12} & m_{22} \\
m_{11}
\end{array}\right)\right\rangle \\
& =\left\{\left[m_{11}-m_{22}\right]_{q}\left[m_{12}-m_{11}+1\right]_{q}\right\}^{1 / 2}\left|\left(\begin{array}{ccc}
m_{13} \cdot m_{23} & m_{33} \\
m_{12} & m_{22} \\
m_{11}-1
\end{array}\right)\right\rangle \\
& F_{\beta}\left|\left(\begin{array}{ccc}
m_{13} & m_{23} & m_{33} \\
m_{12} & m_{22} \\
& m_{11}
\end{array}\right)\right\rangle \\
& =\left\{\frac{\left[m_{12}-m_{11}\right]_{q}\left[m_{13}-m_{12}+1\right]_{q}\left[m_{23}-m_{12}\right]_{q}\left[m_{33}-m_{12}-1\right]_{q}}{\left[m_{12}-m_{22}+1\right]_{q}\left[m_{12}-m_{22}\right]_{q}}\right\}^{1 / 2} \\
& \times\left|\left(\begin{array}{ccc}
m_{13} & m_{23} & m_{33} \\
m_{12}-1 & m_{22} \\
m_{11} &
\end{array}\right)\right\rangle
\end{aligned}
$$




$$
+\left\{\frac{\left[m_{22}-m_{11}-1\right]_{q}\left[m_{13}-m_{22}+2\right]_{q}\left[m_{23}-m_{22}+1\right]_{q}\left[m_{33}-m_{22}\right]_{q}}{\left[m_{12}-m_{22}+2\right]_{q}\left[m_{12}-m_{22}+1\right]_{q}}\right\}^{1 / 2}
$$

(37)

$$
\times\left|\left(\begin{array}{ccc}
m_{13} & m_{2 s} & m_{33} \\
m_{12} & m_{22}-1 \\
& m_{11}
\end{array}\right)\right\rangle
$$

The matrices of $E_{\alpha}$ and $E_{\beta}$ are given by the transposes of $F_{\alpha}$ and $F_{\beta}$, respectively.

For the 6-dimensional representation, we have the following basis vectors:

$$
\begin{array}{ll}
\phi_{1}=\left|\left(\begin{array}{cc}
4 / 3-2 / 3-2 / 3 \\
4 / 3-2 / 3 \\
4 / 3
\end{array}\right)\right\rangle, \quad \phi_{2}=\left|\left(\begin{array}{c}
4 / 3-2 / 3-2 / 3 \\
4 / 3-2 / 3 \\
1 / 3
\end{array}\right)\right\rangle \\
\phi_{3}=\left|\left(\begin{array}{c}
4 / 3-2 / 3-2 / 3 \\
4 / 3-2 / 3 \\
-2 / 3
\end{array}\right)\right\rangle, \quad \phi_{4}=\left|\left(\begin{array}{c}
4 / 3-2 / 3-2 / 3 \\
1 / 3-2 / 3 \\
1 / 3
\end{array}\right)\right\rangle
\end{array}
$$

(38)

$$
\phi_{5}=\left|\left(\begin{array}{c}
4 / 3-2 / 3-2 / 3 \\
1 / 3-2 / 3 \\
-2 / 3
\end{array}\right)\right\rangle, \quad \phi_{6}=\left|\left(\begin{array}{c}
4 / 3-2 / 3-2 / 3 \\
-2 / 3-2 / 3 \\
-2 / 3
\end{array}\right)\right\rangle
$$

Then long computations show that the matrix form of the generators in the 6dimensional representation of $U_{q}\left(A_{2}\right)$ is given by

$$
\begin{aligned}
& h_{\alpha}=\operatorname{diag}(2,0,-2,1,-1,0), \quad h_{\beta}=\operatorname{diag}(0,1,2,-1,0,-2) \\
& E_{\alpha}=[2]_{q}^{1 / 2} e_{12}+[2]_{q}^{1 / 2} e_{23}+e_{45}, \quad F_{\alpha}=[2]_{q}^{1 / 2} e_{21}+[2]_{q}^{1 / 2} e_{32}+e_{54} \\
& E_{\beta}=e_{24}+[2]_{q}^{1 / 2} e_{35}+[2]_{q}^{1 / 2} e_{56}, \quad F_{\beta}=e_{42}+[2]_{q}^{1 / 2} e_{53}+[2]_{q}^{1 / 2} e_{65} \\
& E_{\alpha+\beta}=[2]_{q}^{1 / 2} e_{14}+q e_{25}+[2]_{q}^{1 / 2} e_{46}, \quad F_{\alpha+\beta}=[2]_{q}^{1 / 2} e_{41}+q e_{52}+[2]_{q}^{1 / 2} e_{64} \\
& E_{\beta+n \delta}^{\prime}=q^{-2 n / 3}\left\{q^{-3 n} e_{24}+[2]_{q}^{1 / 2} q^{-3 n} e_{35}+[2]_{q}^{1 / 2} q^{-n} e_{56}\right\} \\
& F_{\beta+n \delta}^{\prime}=q^{2 n / 3}\left\{q^{3 n} e_{42}+[2]_{q}^{1 / 2} q^{3 n} e_{53}+[2]_{q}^{1 / 2} q^{n} e_{65}\right\} \\
& E_{(\delta-\beta)+n \delta}^{\prime}=-q^{-2(n+1) / 3}\left\{q^{-3 n-2} e_{42}+[2]_{q}^{1 / 2} q^{-3 n-1} e_{53}+[2]_{q}^{1 / 2} q^{-n-1} e_{65}\right\} \\
& F_{(\delta-\beta)+n \delta}^{\prime}=-q^{2(n+1) / 3}\left\{q^{3 n+2} e_{24}+[2]_{q}^{1 / 2} q^{3 n+1} e_{35}+[2]_{q}^{1 / 2} q^{n+1} e_{56}\right\} \\
& E_{n \delta}^{\prime(\alpha)}=[2]_{q}^{-1}(-1)^{n-1} \frac{[n]_{q}}{n} q^{-2 n / 3}\left\{\left(q^{n}+q^{-n}\right) e_{11}+q^{-n}\left(q^{2 n}-q^{-2 n}\right) e_{22}\right. \\
&\left.\quad-q^{-2 n}\left(q^{n}+q^{-n}\right) e_{33}+q^{n} e_{44}-q^{-n} e_{55}\right\} \\
& F_{n \delta}^{\prime(\alpha)}=[2]_{q}^{-1}(-1)^{n-1} \frac{[n]_{q}}{n} q^{2 n / 3}\left\{\left(q^{n}+q^{-n}\right) e_{11}-q^{n}\left(q^{2 n}-q^{-2 n}\right) e_{22}\right. \\
&\left.\quad-q^{2 n}\left(q^{n}+q^{-n}\right) e_{33}+q^{-n} e_{44}-q^{n} e_{55}\right\}
\end{aligned}
$$




$$
\begin{aligned}
E_{n \delta}^{\prime}(\beta)=[2]_{q}^{-1} \frac{[n]_{q}}{n} q^{-2 n / 3}\left\{-q^{-2 n} e_{22}-q^{-n}\left(q^{n}+q^{-n}\right) e_{33}+q^{-4 n} e_{44}\right. \\
\left.-q^{-2 n}\left(q^{2 n}-q^{-2 n}\right) e_{55}+q^{-3 n}\left(q^{n}+q^{-n}\right) e_{66}\right\} \\
F_{n \delta}^{\prime(\beta)}=-[2]_{q}^{-1} \frac{[n]_{q}}{n} q^{2 n / 3}\left\{-q^{2 n} e_{22}-q^{n}\left(q^{n}+q^{-n}\right) e_{33}+q^{4 n} e_{44}\right. \\
\left.+q^{2 n}\left(q^{2 n}-q^{-2 n}\right) e_{55}+q^{3 n}\left(q^{n}+q^{-n}\right) e_{66}\right\}
\end{aligned}
$$

We have the following properties for the generators in (39),

$$
\begin{gathered}
\left(E_{\alpha}\right)^{2}=[2]_{q} e_{13},\left(E_{\alpha}\right)^{3}=0,\left(F_{\alpha}\right)^{2}=[2]_{q} e_{31},\left(F_{\alpha}\right)^{3}=0 \\
\left.\left(E_{\alpha+\beta}\right)^{2}=-2\right]_{q} e_{16},\left(E_{\alpha+\beta}\right)^{3}=0,\left(F_{\alpha+\beta}\right)^{2}=-[2]_{q} e_{61},\left(F_{\alpha+\beta}\right)^{3}=0 \\
\left(E_{\beta+n \delta}^{\prime}\right)^{2}=[2]_{q} q^{-5 n-n / 3} e_{36},\left(E_{\beta+n \delta}^{\prime}\right)^{3}=0,\left(F_{\beta+n \delta}^{\prime}\right)^{2}=[2]_{q} q^{5 n+n / 3} e_{63},\left(F_{\beta+n \delta}^{\prime}\right)^{3}=0 \\
\left(E_{(\delta-\beta)+n \delta}^{\prime}\right)^{2}=[2]_{q} q^{-5 n-3-(n+1) / 3} e_{63},\left(E_{(\delta-\beta)+n \delta}^{\prime}\right)^{3}=0 \\
(40) \quad\left(F_{(\delta-\beta)+n \delta}^{\prime}\right)^{2}=[2]_{q} q^{5 n+3+(n+1) / 3} e_{36},\left(F_{(\delta-\beta)+n \delta}^{\prime}\right)^{3}=0
\end{gathered}
$$

as can easily be checked.

Now we are in the position to derive the quantum $R$-matrix, $R_{(3),(6)}(x, y)$, on the tensor product module $V_{(3)} \otimes V_{(6)}$. With the help of (33) and (39) and through long calculations similar to those that led to (34), we find

$$
R_{(3),(6)}(x, y)=f_{q}^{\prime}(x, y) \cdot R_{+} R_{0} R_{-},
$$

where

$$
\begin{aligned}
f_{q}^{\prime}(x, y)= & q^{1 / 3} \cdot \exp \left(\sum_{n>0} \frac{q^{n}-q^{-n}}{q^{2 n}+1+q^{-2 n}} \frac{\left(q^{1 / 3} x / y\right)^{n}}{n}\right) \\
R_{+}=I+ & \frac{y\left(q-q^{-1}\right)}{y-q^{4 / 3} x}\left\{[2]_{q}^{1 / 2}\left(e_{12} \otimes e_{21}+e_{13} \otimes e_{41}+e_{23} \otimes e_{53}\right)+e_{23} \otimes e_{42}\right\} \\
& +\frac{y\left(q-q^{-1}\right)}{y-q^{-2 / 3} x}\left\{e_{12} \otimes e_{54}+[2]_{q}^{1 / 2}\left(e_{12} \otimes e_{92}+e_{13} \otimes e_{64}\right)+q^{-1} e_{13} \otimes e_{52}\right. \\
& \left.+[2]_{q}^{1 / 2} e_{23} \otimes e_{65}\right\}+\frac{y^{2}\left(q-q^{-1}\right)^{2}}{\left(y-q^{4 / 3} x\right)\left(y-q^{-2 / 3} x\right)} e_{13} \otimes e_{52} \\
R_{0}= & \frac{y-q^{-2 / 3} x}{y-q^{4 / 3} x}\left\{e_{11} \otimes e_{11}+e_{22} \otimes e_{33}+e_{33} \otimes e_{66}\right\} \\
& \frac{\left(y-q^{10 / 3} x\right)\left(y-q^{-2 / 3} x\right)}{\left(y-q^{4 / 3} x\right)^{2}}\left\{e_{11} \otimes e_{22}+e_{11} \otimes e_{44}+e_{22} \otimes e_{55}\right\}
\end{aligned}
$$




$$
\begin{aligned}
&+\frac{y-q^{10 / 3} x}{y-q^{4 / 3} x}\left\{e_{11} \otimes e_{33}+e_{11} \otimes e_{55}+e_{11} \otimes e_{66}+e_{22} \otimes e_{66}\right\} \\
&+\frac{y-q^{-2 / 3} x}{y-q^{-8 / 3} x}\left\{e_{22} \otimes e_{11}+e_{33} \otimes e_{11}+e_{33} \otimes e_{22}+e_{33} \otimes e_{33}\right\} \\
&+\frac{\left(y-q^{-2 / 3} x\right)^{2}}{\left(y-q^{4 / 3} x\right)\left(y-q^{-8 / 3} x\right)}\left\{e_{22} \otimes e_{22}+e_{33} \otimes e_{44}+e_{33} \otimes e_{55}\right\} \\
&+\frac{\left(y-q^{10 / 3} x\right)\left(y-q^{-2 / 3} x\right)^{2}}{\left(y-q^{4 / 3} x\right)^{2}\left(y-q^{-8 / 3} x\right)} e_{22} \otimes e_{44} \\
& R_{-}=q\left\{e_{11} \otimes e_{11}+e_{22} \otimes e_{33}+e_{33} \otimes e_{66}\right\}+e_{11} \otimes e_{22}+e_{11} \otimes e_{44} \\
&+e_{22} \otimes e_{22}+e_{22} \otimes e_{55}+e_{33} \otimes e_{44}+e_{33} \otimes e_{55}+q^{-1}\left\{e_{11} \otimes e_{33}\right. \\
&+e_{11} \otimes e_{55}+e_{11} \otimes e_{66}+e_{22} \otimes e_{11}+e_{22} \otimes e_{44} \\
&\left.+e_{22} \otimes e_{66}+e_{33} \otimes e_{11}+e_{33} \otimes e_{22}+e_{33} \otimes e_{33}\right\} \\
&+\frac{x q^{1 / 3}\left(q-q^{-1}\right)}{y-q^{4 / 3} x}\left\{[2]_{q}^{1 / 2}\left(e_{21} \otimes e_{12}+e_{31} \otimes e_{14}+e_{32} \otimes e_{35}\right)+e_{32} \otimes e_{24}\right\} \\
&+\frac{x q^{-2 / 3}\left(q-q^{-1}\right)}{y-q^{-2 / 3} x}\left\{[2]_{q}^{1 / 2}\left(e_{21} \otimes e_{23}+e_{31} \otimes e_{46}\right)+e_{21} \otimes e_{45}+e_{31} \otimes e_{25}+\right.
\end{aligned}
$$

(42)

$$
\left.+[2]_{q}^{1 / 2} e_{32} \otimes e_{56}\right\}+\frac{x^{2} q^{2 / 3}\left(q-q^{-1}\right)^{2}}{\left(y-q^{4 / 3} x\right)\left(y-q^{-2 / 3} x\right)} e_{31} \otimes e_{25} .
$$

It is understood that $I$ in $R_{+}$is the $18 \times 18$ unit matrix.

\section{Concluding Remarks}

To summarise: we have obtained explicit and compact formulae for the quantum $R$-matrices (or the inter-twiners) associated with some interesting representations of $U_{q}\left(A_{2}\right)$. For the case of $V_{(3)} \otimes V_{(6)}$, it is interesting to note in particular the appearance of fractional powers of $q$. It is hoped that these explicit formulae may be useful in physical applications, with associated interesting new features.

Up to now, we have been working in the homogeneous gradation of $U_{q}\left(A_{1}^{(1)}\right)$ and $U_{q}\left(A_{2}^{(1)}\right)$. Similar calculations can be carried out for other gradations, in particular for the principal gradation. In this way, we could get a host of $R$-matrices with different spectral parameter-dependences. The simplest cases corresponding to the fundamental representation of $U_{q}\left(A_{1}\right)$ and $U_{q}\left(A_{2}\right)$ have been worked out very recently [6].

\section{APPENDIX}

We consider here finite-dimensional irreducible representations of $U_{q}\left(g l(n)^{(1)}\right)$ 
with the Chevalley generators $\left\{E_{i}, F_{i}, q^{h_{i}}, 0 \leqslant i<n ; q^{d}\right\}$ in which

$$
E_{i} \equiv E_{i+1}, \quad F_{i} \equiv E_{i+1 i}, \quad q^{h_{i}}, \quad h_{i} \equiv E_{i i}-E_{i+1 i+1}, 1 \leqslant i<n, \quad q^{E_{n n}}
$$

are the usual Chevalley generators of $U_{q}(g l(n))$. We define

$$
\begin{aligned}
& E_{i j}=E_{i k} E_{k j}-q^{-1} E_{k j} E_{i k}, \quad i<k<j \\
& E_{i j}=E_{i k} E_{k j}-q E_{k j} E_{i k}, \quad i>k>j
\end{aligned}
$$

and put

$$
E_{\psi} \equiv q^{E_{11}+E_{n n}} E_{1 n}, \quad F_{\psi} \equiv E_{n 1} q^{-E_{11}-E_{n n}}, \quad h_{\psi} \equiv E_{11}-E_{n n}
$$

Then we have

Proposition. For any given $z \in \mathbf{C}^{\times}$, there is a homomorphism of algebras $\mathrm{ev}_{z}: U_{q}\left(g l(n)^{(1)}\right) \rightarrow U_{q}(g l(n))$ where, in terms of the Chevalley generators,

$$
\begin{aligned}
& \mathrm{ev}_{z}\left(E_{i}\right)=E_{i}, \quad \mathrm{ev}_{z}\left(F_{i}\right)=F_{i}, \quad \mathrm{ev}_{z}\left(h_{i}\right)=h_{i} \\
& \mathrm{ev}_{z}\left(E_{0}\right)=z F_{\psi}, \quad \operatorname{ev}_{z}\left(F_{0}\right)=z^{-1} E_{\psi}, \quad \mathrm{ev}_{z}\left(h_{0}\right)=-h_{\psi}, \quad \mathrm{ev}_{z}(c)=0
\end{aligned}
$$

ProOF: To show that a homomorphism $U_{q}\left(g l(n)^{(1)}\right) \rightarrow U_{q}(g l(n))$ is defined, one needs to check that the relations in (1) are satisfied. This is immediate except for the last two, which reduce to

$$
\begin{aligned}
& \left(\operatorname{ad}_{q^{-1}} F_{i}\right)^{1+\left(\psi, \alpha_{i}\right)} F_{0}=\left(\operatorname{ad}_{q^{-1}} F_{i}\right)^{1+\left(\psi, \alpha_{i}\right)} E_{\psi}=0 \\
& \left(\operatorname{ad}_{q^{-1}} F_{0}\right)^{1+\left(\psi, \alpha_{i}\right)} F_{i}=\left(\operatorname{ad}_{q^{-1}} E_{\psi}\right)^{1+\left(\psi, \alpha_{i}\right)} F_{i}=0
\end{aligned}
$$

and two similar relations with the interchanges $F_{i} \leftrightarrow E_{i}, F_{0} \leftrightarrow E_{0}, q^{-1} \leftrightarrow q$. We now prove the result (47). First we consider the case: $1<i<i+1<n$. In this case $\left(\psi, \alpha_{i}\right)=0$, and the left hand side of (47) becomes

$$
\left(\operatorname{ad}_{q^{-1}} F_{i}\right) E_{\psi}=\left[F_{i}, E_{\psi}\right]=q^{E_{11}+E_{n n}}\left[E_{i+1 i}, E_{1 n}\right]
$$

which can easily be seen to vanish. We then consider the $i=1$ case. Then the left hand side of (47) reads

$$
\left(\operatorname{ad}_{q^{-1}} F_{1}\right)^{2} E_{\psi}=\left(\operatorname{ad}_{q^{-1}} E_{21}\right)\left(\operatorname{ad}_{q^{-1}} E_{21}\right) E_{\psi}
$$

One can easily show that $\left(\operatorname{ad}_{q^{-1}} E_{21}\right) E_{\psi}=q^{E_{22}+E_{n n}} E_{2 n}$. Inserting this into (50), one sees that this equation reduces to

$$
\left(\operatorname{ad}_{q-1} E_{21}\right) q^{E_{22}+E_{n n}} E_{2 n}=q^{E_{22}+E_{n n-1}}\left[E_{21}, E_{2 n}\right]=0
$$


as required. Finally for $i=n$, we see that the left hand side of (47) reduces to

$$
\left(\operatorname{ad}_{q^{-1}} F_{n}\right)^{2} E_{\psi}=\left(\operatorname{ad}_{q^{-1}} E_{n n-1}\right)\left(\operatorname{ad}_{q^{-1}} E_{n n-1}\right) E_{\psi} .
$$

Direct computations show that the right hand side of this equation reduces to

$$
q^{E_{11}+E_{n n-1}}\left\{q^{-1} E_{n n-1}\left[E_{n n-1}, E_{1 n}\right]-q\left[E_{n n-1}, E_{1 n} E_{n n-1}\right\}\right.
$$

which, using the formula

$$
\left[E_{n n-1}, E_{1 n}\right]=-q^{E_{n-1 n-1}-E_{n n}} E_{1 n-1},
$$

is easily seen to vanish. We may prove (48) similarly.

REMARK. Since $N \equiv \sum_{i=1}^{n} E_{i i}$ commutes with all generators, it follows that if we set, instead of (45),

$$
E_{\psi} \equiv q^{E_{11}+E_{n n}-\frac{2}{n} N} E_{1 n}, \quad F_{\psi} \equiv E_{n 1} q^{-E_{11}-E_{n n}+\frac{2}{n} N} E_{1 n},
$$

then the proposition of this Appendix still holds. It turns out that it is more convenient to use (55) as we did in the previous sections.

\section{REFERENCES}

[1] L. Alvarez-Gaumé, C. Gomez and G. Sierra, 'Quantum group interpretation of some conformal field theories', Phys. Lett. B 220 (1989), 142-152.

[2] L. Alvarex-Gaumé, C. Gomez and G. Sierra, 'Hidden quantum symmetries in rational conformal field theories', Nuclear Phys. B 319 (1989), 155-186.

[3] O. Babelon and L. Bonora, 'Conformal affine $s l_{2}$ Toda field theory', Phys. Lett. B 244 (1990), 220-226.

[4] R.J. Baxter, Exactly solved models in statistical mechanics (Academic Press, New York, 1982).

[5] A.J. Bracken, G.W. Delius, M.D. Gould and Y.-Z. Zhang, 'Solutions to the (graded) Yang-Baxter equation with extra non-additive parameterst', J. Phys. A. (to appear).

[6] A.J. Bracken, G.W. Delius, M.D. Gould and Y.-Z. Zhang, 'Infinite families of gaugeequivalent $\mathrm{R}$-matrices and gradations of quantized affine algebras', Internat. J. Modern Phys. (to appear).

[7] G.W. Delius, M.D. Gould and Y.-Z. Zhang, 'On the construction of trigonometric solutions of the Yang-Baxter equation', Nuclear Phys. B (to appear).

[8] V.G. Drinfeld, 'Quantum groups', Proc. ICM, Berkeley 1 (1986), 798-820.

[9] L.D. Faddeev, 'Integrable models in (1+1)-dimensional quantum field theory', in Recent advances in field theory and statistical mechanics (North-Holland, New York, 1984), pp. 563-608. 
[10] M. Jimbo, 'Ag-difference analogue of $U(g)$ and the Yang-Baxter equation', Lett. Math. Phys. 10 (1985), 63-69.

[11] M. Jimbo, 'A $q$-analogue of $U(g l(N+1))$ Hecke algebra and the Yang-Baxter equation', Lett. Math. Phys. 11 (1986), 247-252.

[12] M. Jimbo, 'Quantum $R$ matrix for the generalized Toda system', Comm. Math. Phys. $102(1986)$, 537-542.

[13] V.F.R. Jones, 'Baxterization', Internat. J. Modern Phys. B 4 (1990), 701-713.

[14] V.G. Kac, Infinite dimensional Lie algebras: An introduction (Birkhäuser, Boston, 1983).

[15] S.M. Khoroshkin and V.N. Tolstoy, 'The uniqueness theorem for the universal $R$-matrix', Lett. Math. Phys. 24 (1992), 231-244.

[16] S.M. Khoroshkin and V.N. Tolstoy, 'The universal $R$-matrix for quantum non-twisted affine Lie algebras', Funktsional. Anal. i Prilozhen 26 (1992), 85-88.

[17] A.N. Kirillov and N. Reshetikhin, 'Representations of the algebra $U_{q}(s l(2))$, $q$-orthogonal polynomials and invariants of links', preprint LOMI E-9-88.

[18] J.R. Links, M.D. Gould and R.B. Zhang, 'Quantum supergroups, link polynomials and representations of the braid generator', Rev. Math. Phys. 5 (1993), 345-361.

[19] G. Moore and N.Yu. Reshetikhin, 'A comment on quantum group symmetry in conformal field theory', Nuclear Phys. B 328 (1989), 557-574.

[20] N. Reshetikhin, 'Quantized universal enveloping algebras, the Yang-Baxter equation and inveriants of links: I, II', preprints LOMI E-4-87, E-17-87.

[21] F. Toppan and Y.-Z. Zhang, 'Superconformal affine Lionville theory', Phys. Lett. B 292 (1992), 67-76.

[22] M. Wadati, T. Deguchi and Y. Akutsu, 'Exactly solvable models and knot theory', Phys. Rep. 180 (1989), 247-332.

[23] E. Witten, 'Quantum field theory and the Jones polynomial', Commun. Math. Phys. 121 (1989), 351-399.

[24] R.B.Zhang, M.D.Gould and A.J.Bracken, 'Quantum group invariants and link polynomials', Commun. Math. Phys. 137 (1991), 13-27.

[25] R.B. Zhang, M.D. Gould and A.J. Bracken, 'From representations of the braid group to solutions of the Yang-Baxter equation', Nuclear Phys. B 354 (1991), 625-652.

[26] Y.-Z. Zhang and M.D. Gould, 'Quantum affine algebras and universal $R$-matrix with spectral parameter', Lett. Math. Phys. 31 (1994), 101-110.

[27] Y.-Z. Zhang and M.D. Gould, 'On universal $R$-matrix for quantized nontwisted rank 3 affine $K M$ algebras', Lett. Math. Phys. 29 (1993), 19-31.

[28] Y.Z. Zhang and M.D. Gould, 'Unitarity and complete reducibility of certain modules over quantized affine Lie algebras', J. Math. Phys. 34 (1993), 6045-6059.

\footnotetext{
Department of Mathematics

University of Queensland

Queensland 4072

Australia
} 